\title{
Veno-Venous Extracorporeal Membrane Oxygenation for Fat Embolism
}

\author{
Kamen Valchanov ${ }^{\mathrm{a}, \mathrm{e}}$, Ari Ercole ${ }^{\mathrm{b}}$, Jo-anne Fowles ${ }^{\mathrm{a}}$, \\ Jas Parmarc, Deepa Gopalan ${ }^{\mathrm{d}}$
}

\begin{abstract}
Pulmonary fat embolism is an infrequent but a serious complication of major trauma. Patients can suffer severe respiratory failure with sudden and unexpected onset, which can happen within 24 hours of trauma. The spectrum of presentations can vary from mild shortness of breath to sudden death. When respiratory failure ensues the clinical and radiological presentation resembles acute respiratory distress syndrome (ARDS). There is no evidence-based specific treatment for the condition, and conventional respiratory support often leads to recovery. In extreme cases the resulting hypoxemia and hypercarbia can be fatal. In other ARDS cases temporary support with extracorporeal membrane oxygenation (ECMO) can offer $65 \%$ survival (ELSO registry data). However, in cases of fat embolism the fat load can be transferred to the oxygenator and impact its function, and there is a scarcity of published data, leading to a relative reluctance to provide such support. We present a case of a 32-year-old patient following from traumatic bilateral below knee amputations. He suffered from pulmonary fat embolism leading to ARDS, which could not be adequately supported by conventional mechanical ventilation of the lungs. We considered the disadvantages of oxygenator malfunction due to fat loading, as well as the need for early oxygenator replacement. However, on the grounds of good prognosis for recovery, and potential for a short term ECMO support, the decision was made to commence veno-venous ECMO. Support on VV ECMO for total of 6 days allowed successful respiratory recovery and subsequent discharge home with ongoing rehabilitation.
\end{abstract}

\footnotetext{
Manuscript accepted for publication July 24, 2014

${ }^{\mathrm{a}}$ Department of Anesthesia and Intensive Care, Papworth Hospital, Cambridge CB23 3RE, UK

${ }^{\mathrm{b}}$ University of Cambridge Division of Anesthesia, Box 93

Addenbrooke's Hospital, Hills Road, Cambridge CB2 2QQ, UK

${ }^{\mathrm{c}}$ Department of Transplantation, Papworth Hospital, Cambridge CB23 3RE, UK

${ }^{\mathrm{d}}$ Department of Radiology, Papworth Hospital, Cambridge CB23 3RE, UK

${ }^{\mathrm{e}}$ Corresponding Author: Kamen Valchanov, Department of Anesthesia and Intensive Care, Papworth Hospital, Cambridge CB23 3RE, UK.

Email: kamen.valchanov@papworth.nhs.uk
}

doi: http://dx.doi.org/10.14740/jmc1877e
Keywords: Fat embolism; Major trauma; Respiratory failure; ARDS; Extracorporeal oxygenation; ECMO

\section{Introduction}

Pulmonary fat embolism can lead to potentially fatal respiratory failure presenting as rapidly developing acute respiratory distress syndrome (ARDS). The spectrum of presentations can vary from mild shortness of breath to sudden death [1]. When respiratory failure ensues the clinical and radiological presentation resembles ARDS due to any other pathology [2]. In the majority of cases the respiratory function can be supported by conventional mechanical ventilation. In cases where despite mechanical ventilation the gas exchange is so poor that the patient may die imminently, extracorporeal membrane oxygenation (ECMO) support can be offered. However, the potential for fat deposits seeding in the oxygenator and impairing its function, as well as scarcity of published cases, leads to reluctance to use this support. On the other hand, the course of ECMO support is unlikely to be prolonged in previously fit patients, and a simple early replacement of the oxygenator, if necessary, can lead to a successful outcome. We present a successful veno-venous ECMO support for a previously fit patient suffering from near-fatal pulmonary fat embolus as a result of major trauma.

\section{Case Report}

A previously fit 32-year-old male patient was involved in a road traffic accident as a pedestrian, sustaining traumatic lower limb amputations. GCS was 15 at the scene. He was transferred to a tertiary trauma center where he underwent debridement and formalization of the amputations. No other skeletal injuries were found, and only minor bruises were evident. The admission trauma chest X-ray was not indicative of trauma or pre-existing lung disease (Fig. 1). Following completion of surgery the patient woke up, and the trachea was extubated. The gas exchange was normal in the recovery room, and supplemental $28 \%$ oxygen via nasal cannulae was 


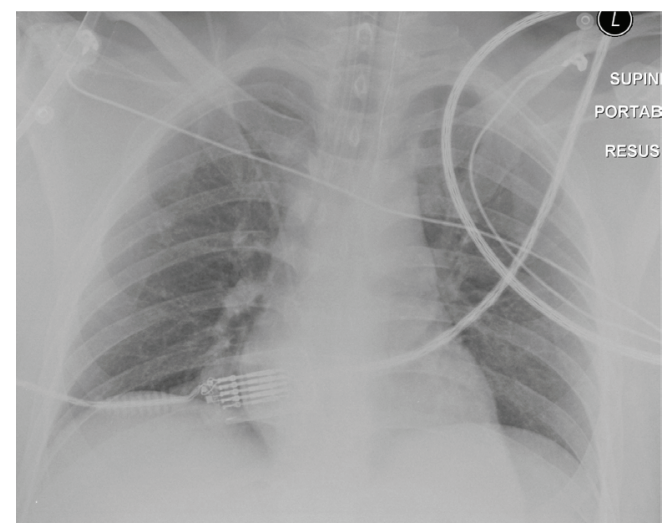

Figure 1. A rotated supine chest radiograph on admission demonstrates clear lungs and pleural spaces.

used, producing oxygen saturations above $95 \%$.

Within $2 \mathrm{~h}$ of surgery, the gas exchange suddenly deteriorated, and emergency intubation and mechanical ventilation of the lungs was re-instituted. The chest X-ray demonstrated bilateral widespread reticular shadowing, consistent with fat embolism. Low tidal volume ventilation, moderately high PEEP (12 $\mathrm{mm} \mathrm{Hg}$ ), muscle relaxation, and negative fluid balance via hemofiltration and intravenous antibiotics were instituted as a ventilatory protective strategy bundle. Cardiogenic pulmonary edema was ruled out by echocardiography demonstrating normal left and right ventricular function. The diagnosis of fat embolus was one of exclusions due to a sug-

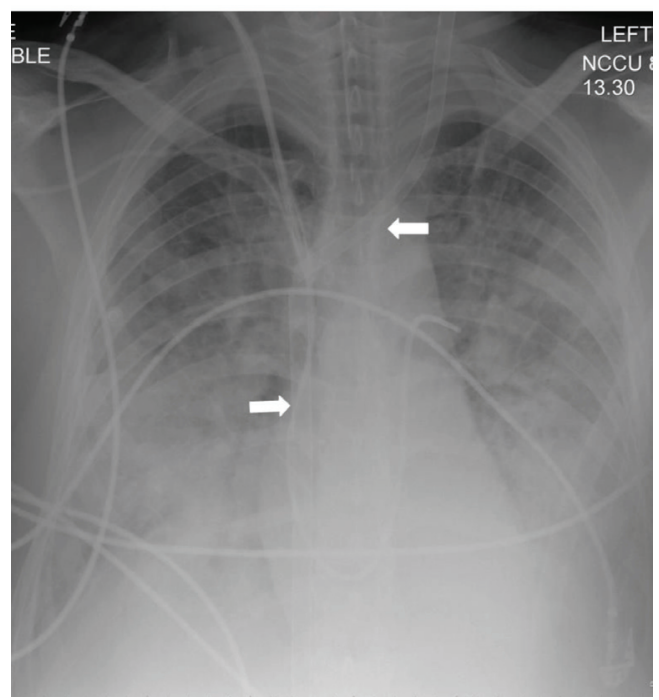

Figure 2. Supine chest radiograph over the next $12 \mathrm{~h}$ showed progressive worsening in the bilateral alveolar opacification predominantly involving the mid- and lower zones and development of small to moderate pleural effusions. Note the presence of the veno-venous ECMO cannulae (block arrows). Despite the lack of recirculation of oxygenated blood the IVC cannula was withdrawn by $5 \mathrm{~cm}$.

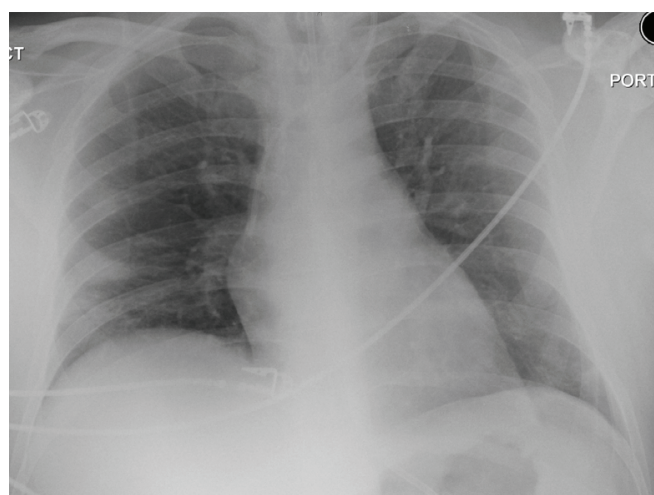

Figure 3. Allowing for the rotation, the AP chest radiograph post decannulation demonstrates complete resolution of the parenchymal opacification and pleural effusions.

gestive chest X-ray following major trauma and lower limb surgery, as well as absence of other risk factors. The patient's clinical condition did not improve, and the gas exchange deteriorated further with $\mathrm{pH} 7.02, \mathrm{PO}_{2} 7 \mathrm{kPa}, \mathrm{PaCO}_{2} 8.75 \mathrm{kPa}$, on $\mathrm{FIO}_{2}$ 1, BE-16 and Lac-11.1. The deterioration was radiologically confirmed with X-ray consistent with ARDS.

The local ECMO center was contacted and a team mobilized to assess the patient. The previously published cases of patients with fat embolism supported by ECMO were reviewed, and negative experiences with fat degrading the oxygenator function were noted $[3,4]$. As this was a young patient with no previous co-morbidities, removed potential source of fat embolism, and an expected short ECMO run, the team decided to offer extracorporeal support, and consider early oxygenator replacement if required.

Peripheral mobile veno-venous ECMO was instituted in the referring hospital. Left femoral vein was cannulated with a 25G $55 \mathrm{~cm}$ Macquet Ven cannula for venous drainage, connected to a heparin bonded circuit with Medos oxygenator, and return Macquet Art 19G $15 \mathrm{~cm}$ cannula though the left internal jugular vein for venous return (Fig. 2). Oxygenation improved immediately, and ventilatory support was reduced to the protective levels. The patient was then transferred to the local ECMO center. After 6 days of ECMO support the patient was decannulated, the trachea was extubated on the following day (Fig. 3). He was transferred back to the trauma center for further convalescence.

Whilst ECMO supported oxygenation and $\mathrm{CO}_{2}$ removal, the performance of the oxygenator was tested daily with a view of early replacement. Although the degradation of oxygen flux was slightly faster than usual in this case, it was sufficient for a 6-day run without changing the oxygenator. The $\mathrm{PO}_{2}$ output from the oxygenator was $69.8 \mathrm{kPa}$ on day 1 , and gradually declined to $55.8,38.9,44.5,49$, and 39.6 $\mathrm{kPa}$ on day 5 (before ECMO removal), all of which provided adequate oxygenation (Fig. 4). At the time of ECMO cannulation use of a dual lumen Avalon Elite cannula was con- 


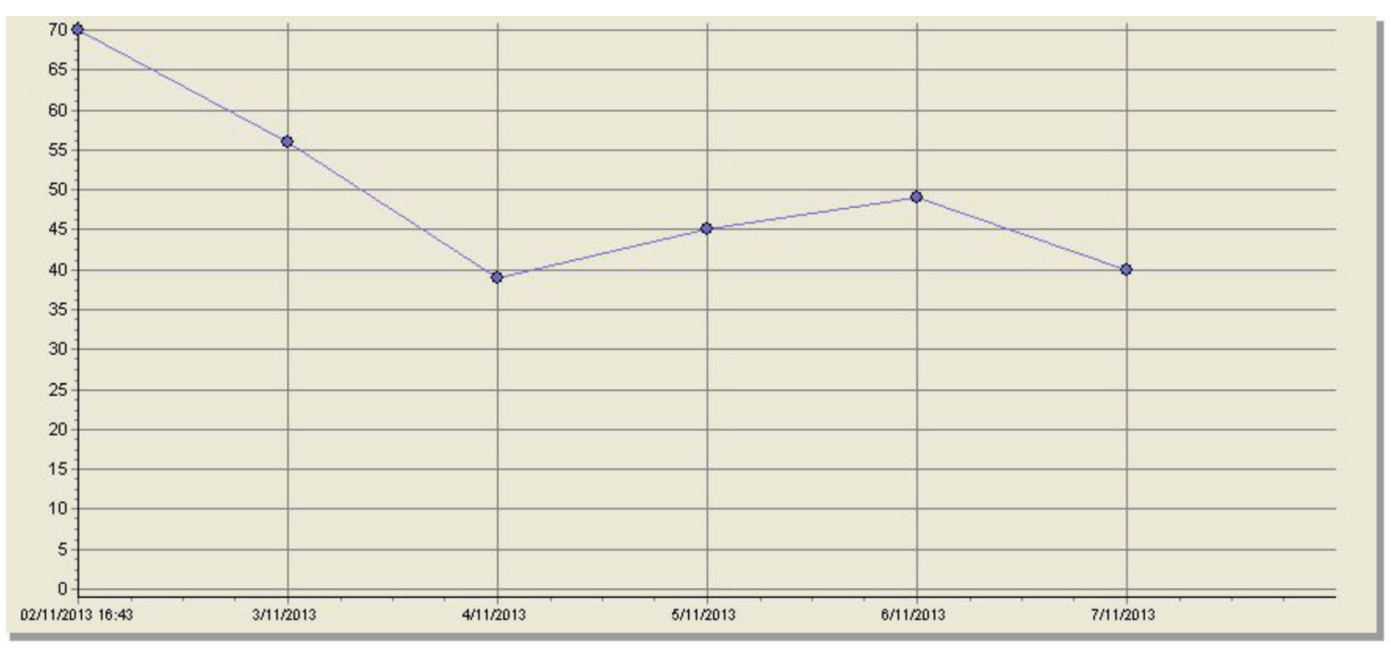

Figure 4. Oxygenator output: $\mathrm{x}$-axis: days on $\mathrm{ECMO}$; y-axis: $\mathrm{PO}_{2}$ of blood post oxygenator.

sidered, but due to limitation of flow, a single lumen drainage cannula was chosen in an attempt to offer better venous drainage, and higher flows, in case lower oxygen output from the oxygenator needed to be temporarily tolerated.

Phantom limb pain was anticipated and treatment with prophylactic gabapentin and ketamine was instituted within $24 \mathrm{~h}$ of amputations. The patient continued to make a good recovery and was discharged home with a rehabilitation program in progress. At a follow-up outpatient visit at the ECMO center the patient was found to have made excellent physical recovery, with early mobilization, and gradually reducing phantom limb pain.

\section{Discussion}

In the case we present, we utilized a rapidly expanding technology for support of respiratory failure by extracorporeal oxygenation. This modality of respiratory support has not been frequently reported for fat embolus cases. Indeed the performance of the oxygenator used degrades once polluted with the embolic fat material, which could be aspirated from the pulmonary vascular bed during drainage of venous blood into the ECMO circuit. However, when the source of fat embolism has been removed, in this case by traumatic amputations, a simple replacement of the oxygenator is likely to resolve the problem. We noticed a reduction in the oxygen output over the days but in fact the systemic oxygenation remained adequate and we did not need to replace the device.

A second lesson from this case is that the use of extracorporeal support is increasingly being used for respiratory and circulatory support in trauma cases. The recent advances in technology and greater clinical experience have made ECMO safer and more popular. The majority of patients are in previous good health and are unlikely to need prolonged extracorporeal support. Even when prolonged support is required, the ECMO circuit could be used as a bridge to organ transplantation.

Finally, in complex cases where trauma leads to long term disability and pain, the surgical and intensive care teams need to address the problem holistically considering the impact that early interventions may have on the quality of life of the patient and their family.

\section{Grant Support}

None.

\section{Conflict of Interest}

None.

\section{References}

1. Akhtar S. Fat embolism. Anesthesiol Clin. 2009;27(3):533-550, table of contents.

2. Arora S, Singh PM, Trikha A. Ventilatory strategies in trauma patients. J Emerg Trauma Shock. 2014;7(1):25-31.

3. Maunz O, Penn S, Simon A. Emergency oxygenator change-out after massive fat embolism. Perfusion. 2013;28(2):167-169.

4. Webb DP, McKamie WA, Pietsch JB. Resuscitation of fat embolism syndrome with extracorporeal membrane oxygenation. J Extra Corpor Technol. 2004;36(4):368370 . 